\title{
Bilateral Nephrectomy in Patients with Autosomal Dominant Polycystic Kidney Disease and End-Stage Chronic Renal Failure
}

\author{
Alexander E. Lubennikov ${ }^{a}$ Nicolay V. Petrovskii ${ }^{b} \quad$ German E. Krupinov ${ }^{b}$ \\ Evgeniy M. Shilov ${ }^{c}$ Roman N. Trushkin ${ }^{a}$ Oleg N. Kotenko ${ }^{a}$ Petr V. Glybochkob \\ ${ }^{a}$ Municipal Clinical Hospital No. 52 of the Moscow Department of Healthcare, Moscow, Russian Federation; \\ bInstitute of Urology and Human Reproductive Health, First Moscow State Medical University (Sechenov University), \\ Moscow, Russian Federation; 'Clinic of Rheumatology, Nephrology and Occupational Pathology named after E.M. \\ Tareev, First Moscow State Medical University (Sechenov University), Moscow, Russian Federation
}

\section{Keywords}

Autosomal dominant polycystic kidney disease · End-stage kidney disease - Bilateral nephrectomy

\begin{abstract}
Background: In patients with autosomal dominant polycystic kidney disease (ADPKD) and end-stage kidney disease, bilateral nephrectomy $(\mathrm{BN})$ is currently performed predominantly via the laparoscopic approach. We analysed the results of BN depending on the approach and preoperative and perioperative factors. Patients and Methods: This was a single-centre retrospective study carried out from April 2010 to March 2020, including a total of 142 patients presenting with ADPKD who were treated by BN. Of these, 108 patients meeting the inclusion criteria were selected to analyse the results. We compared therapeutic outcomes depending on the surgical approach (laparotomy or laparoscopy) and the type of the operation (emergent or elective). Results: Of the 108 eligible patients, 36 (group I) underwent laparoscopic $\mathrm{BN}$ and the remaining 72 patients (group II) were subjected to midline laparotomy. Sixty-nine patients underwent elective surgery and 39 endured emergent operations. The most frequent indications (87 patients, $80.6 \%$ ) for surgical treatment were urinary tract infection and infected cysts. The me-
\end{abstract}

dian length of hospital stay for group I and group II patients amounted to 8 days (IQR: 7.5-9) and 12.5 days (IQR: 9-16.5), respectively ( $p<0.001$ ). However, comparing the patients operated on electively, the actual difference in the length of hospital stay was inconsiderable: median 8 days (IQR: 7-9) in group I and 9 days (IQR: 9-11.5) in group II. The median duration of the operation was significantly $(p<0.001)$ longer in group I amounting to 217.5 min (IQR: 197.5-305) than in group II equalling $115 \mathrm{~min}$ (IQR: 107.5-145). The frequency of postoperative complications, lethal outcomes, and blood loss volume did not statistically significantly differ depending on the surgical approach. Only patients operated on emergency underwent releparotomy due to intraoperative large bowel injury. Lethal outcomes ( $n=18,16.7 \%)$ after surgery were observed only in emergent patients. Sepsis prior to surgery, systemic inflammation response syndrome (SIRS) with the CRP level above $173 \mathrm{mg} / \mathrm{mL}$, prolonged preoperative antibacterial therapy, and undiagnosed large bowel injury were associated with a lethal outcome after BN. Conclusion: The results of open and laparoscopic BN in elective surgery were comparable. Emergency operations for infected renal cysts and SIRS were associated with increased incidence of large bowel injury and lethal outcomes.

(C) 2021 The Author(s)

Published by S. Karger AG, Basel karger@karger.com www.karger.com/nef

Karger $\stackrel{\text { ' }}{5}$

BOPEN ACCESS
(C) 2021 The Author(s)

Published by S. Karger AG, Basel

This is an Open Access article licensed under the Creative Commons Attribution-NonCommercial-4.0 International License (CC BY-NC) (http://www.karger.com/Services/OpenAccessLicense), applicable to the online version of the article only. Usage and distribution for commercial purposes requires written permission.
Nicolay V. Petrovskii

Institute of Urology and Human Reproductive Health

First Moscow State Medical University (Sechenov University)

Bol'shaya Pirogovskaya Ulitsa, Moscow 119146 (Russian Federation)

n_petrovskiy@hotmail.com 


\section{Introduction}

The main indications for bilateral nephrectomy for autosomal dominant polycystic kidney disease (ADPKD) in patients with end-stage kidney disease (ESKD) include abdominal symptoms (pain and early satiety) due to massively enlarged kidneys, need for space for future transplant, infected cysts, relapsing macrohaematuria, suspected renal cancer, and nephrogenic arterial hypertension [1]. Historically, open bilateral nephrectomy (BN) performed through the midline laparotomy has been associated with a high rate of postoperative complications and high risk of a lethal outcome. Thus, Bennet et al. [2], as early as in 1973, reported a high rate of severe complications (38\%) and mortality (3\%). Mendelssohn et al. [3] in 1988 confirmed these data, reporting the results of treating a total of 35 patients, with the postoperative mortality amounting to $5 \%$. Another problem of open $\mathrm{BN}$ is high frequency of blood transfusion which may lead to sensitization of the recipient and difficulties with selection of the donor kidney. Publications of recent years have demonstrated significantly more frequent need for haemotransfusion in open $\mathrm{BN}$ as compared with a laparoscopic intervention $[4,5]$.

Currently, laparoscopic BN for ADPKD is an effective and safe procedure in the majority of cases. However, there exist problems of removing very large kidneys. Lipke et al. [6] reported an increased incidence of conversion from laparoscopic to open surgery in patients with kidneys of a volume larger than 3,500 $\mathrm{mL}$ in their series of 18 patients. Furthermore, Ivey et al. [7] showed that larger kidneys were not only associated with conversion to open surgery with a mass greater than $1,500 \mathrm{~g}$ but also had longer operative times and increased blood loss, as well as increased risk of conversion. Some surgeons in order to facilitate kidney extraction and to shorten the postoperative scar proposed to aspirate the cysts intracorporeally to morcellate the kidney $[8,9]$; however, these techniques did not receive wide implementation because they were associated with an elevated risk for the development of peritonitis and dynamic intestinal obstruction $[10,11]$.

The use of hand-assisted laparoscopic BN makes it possible to decrease severity of postoperative complications and duration of laparoscopic nephrectomy $[12,13]$. However, removal of very large kidneys requires extension of the hand-port incision, thus interfering with otherwise acceptable cosmesis after a minimally invasive operation; moreover, a series of publications reported an increased incidence of postoperative hernias $[5,12]$.
These aspects encouraged us to carry out a detailed analysis of the results of open BN performed via the midline laparotomic approach compared with laparoscopic BN.

\section{Patients and Methods}

Between April 2010 and March 2020, BN was performed in a total of 142 patients. Of these, 34 patients were excluded from the study because of accompanying septic pathology: catheter-associated bloodstream infection, suppuration of hepatic cysts, dialysis-related peritonitis, prostatitis, and a recurrent course of pyelonephtitis of the transplanted kidney. Hence, the results of operative treatment were assessed in 108 eligible patients. Group I included 36 patients subjected to laparoscopic BN, and group II consisted of 72 patients undergoing open BN. The main characteristics of patients and indications for surgery in the 2 groups are shown in Table 1.

The most frequent indications for BN were as follows: urinary tract infection, infected renal cysts, or an active course of pyelonephritis. In the overwhelming majority of group I patients $(n=33$, $91.7 \%$ ), laparoscopic BN was performed electively, whereas open $\mathrm{BN}$ in $50 \%$ of patients was performed for emergency indications defined as the presence of infected renal cysts with the systemic inflammation response syndrome (SIRS) [14] or sepsis. The overwhelming majority of emergency patients, prior to operation, had received antibacterial therapy at another hospital, however, with no success due to exhausted therapeutic reserves, and minimally invasive interventions (percutaneous drainage of renal cysts) were impossible to perform due to multiple bilateral infected cysts. Nine patients at the time of surgical intervention had sepsis. In all cases, the reserves of conservative methods of treatment were exhausted. The patients during averagely $14 \pm 3$ days had received antibacterial therapy with de-escalation by the results of urine or blood cultures and extracorporeal methods of detoxification, however, with no positive dynamics.

Prior kidney transplantation did not influence the choice of the operation. Performing only the laparoscopic approach was determined by the lack of SIRS and an elective type of the operation.

The open operation was performed via the midline laparotomic approach. During the operation in all cases, we used a computercontrolled electrosurgical unit ("LigaSure," Switzerland). Laparoscopic nephrectomy was carried out according to the classical technique alternately from each side with rotation of the patient to the lateral position. Removal of the kidneys during laparoscopy was carried out either from the midline approach in the epigastrium or from the transverse suprapubic approach. The length of the incision in laparotomy was chosen depending on the length of the kidney and that in laparoscopy on the kidney diameter measured on MRI scans.

The obtained data were statistically processed using the SPSS version 26 (IBM, Armonk, NY, USA). The quantitative data were not normally distributed, the grouping parameter was always binary, in which connection the Mann-Whitney U test was used. The nominal data were analysed using Fisher's exact test or Pearson $\chi^{2}$. Differences were regarded as statistically significant if $p<0.05$. The effect size of nominal variables was assessed using Cramer's V criterion. The effect size was considered strong if $V>0.4$ according to the recommendations of Rea and Parker [15]. ROC curve analysis was performed to assess diagnostic efficacy of the CRP level in 
Table 1. Main characteristics of patients and indications for surgery

\begin{tabular}{lcc}
\hline Characteristic & $\begin{array}{c}\text { Laparoscopic BN } \\
(n=36) \text {, group I }\end{array}$ & $\begin{array}{l}\text { Open BN } \\
(n=72) \text {, group II }\end{array}$ \\
\hline Age, years & 53.5 (IQR: 43.5-57.5) & 55.5 (IQR: 48.5-60.5) \\
Men, $n(\%)$ & $21(58.3)$ & $39(54.2)$ \\
Women, $n(\%)$ & $15(41.7)$ & $33(45.8)$ \\
Presence of transplanted kidney, $n(\%)$ & $12(33.3)$ & 0 \\
Infected cysts and pyelonephritis, $n(\%)$ & $27(75)$ & $60(83.3)$ \\
Pain and abdominal symptoms, $n(\%)$ & $6(16.7)$ & $12(15.7)$ \\
Macrohaematuria, $n(\%)$ & $3(8.3)$ & 0 \\
Emergency operation, $n(\%)$ & $3(8.3)$ & $36(50)$ \\
\hline
\end{tabular}

BN, bilateral nephrectomy.

Table 2. Comparison of the main perioperative and postoperative data in groups I and II

\begin{tabular}{|c|c|c|c|}
\hline Parameter & $\begin{array}{l}\text { Laparoscopic BN } \\
(n=36) \text {, group I }\end{array}$ & $\begin{array}{l}\text { Open BN } \\
(n=72) \text {, group II }\end{array}$ & $p$ value \\
\hline Volume of blood loss, $\mathrm{mL}$ & 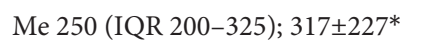 & Me 300 (IQR 200-500); 367士225 & 0.048 \\
\hline Duration of operation, min & 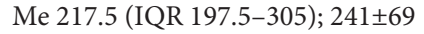 & Me 115 (IQR 107.5-145); 127士32 & $<0.001$ \\
\hline Length of hospital stay, days & Me 8 (IQR 7.5-9); 9 2 & Me 12.5 (IQR 9-16.5); $15 \pm 8$ & $<0.001$ \\
\hline Length of postoperative incision, $\mathrm{cm}$ & Me 15 (IQR 13.5-15); $14 \pm 1$ & Me 22 (IQR 22-24); 23 \pm 2 & $<0.001$ \\
\hline $\begin{array}{l}\text { Prolonged antibacterial therapy ( } 2 \text { weeks and more) prior to } \\
\text { operation, }{ }^{* *} n(\%)\end{array}$ & $12(33.3)$ & $27(37.5)$ & 0.67 \\
\hline Sepsis before operation, $n(\%)$ & 0 & $9(12.5)$ & 0.028 \\
\hline Intraoperative haemorrhage, frequency, $n(\%)$ & $3(8.3)$ & $3(4.2)$ & 0.39 \\
\hline Intestinal injury, frequency, $n(\%)$ & $3(8.3)$ & $3(4.2)$ & 0.39 \\
\hline Relaparatomy, frequency, $n(\%)$ & $3(8.3)$ & $6(8.3)$ & 1.0 \\
\hline Lethal outcome in early postoperative period, frequency, $n(\%)$ & $3(8.3)$ & $15(20.8)$ & 0.17 \\
\hline
\end{tabular}

BN, bilateral nephrectomy; Me, median. * Quantitative parameters for clarity are expressed as medians with IQRs and means with standard deviation. ** Does not include administration of trimethoprim-sulfamethoxazole after kidney transplantation.

prediction of postoperative mortality, as well as to determine the optimal classification threshold ("cut-off" point). In order to reveal the dependence of postoperative lethality on the quantitative and qualitative parameters, we carried out binary logistic regression using the Wald backward elimination technique.

\section{Results}

Comparing the 2 groups depending on the kidney length demonstrated statistically significant differences $(p<0.001)$, but the actual difference was inconsiderable. In the first group, the median of the kidney length amounted to $23 \mathrm{~cm}$ (IQR: 20-25); in the second group, 26 cm (IQR: 24-30). The kidney length was measured during description of the gross specimen. The results of comparing the main perioperative and postoperative data are shown in Table 2.
Laparoscopic BN was characterized by lesser intraoperative blood loss; however, the actual difference was inconsiderable, amounting to $50 \mathrm{~mL}$, by a shorter length of hospital stay and substantially longer time of surgical intervention (difference of medians $102.5 \mathrm{~min}$ ). The average time to flip the patient in the laparoscopic approach amounted to $20 \pm 10 \mathrm{~min}$.

Severe intraoperative complications were observed in $16(14.8 \%)$ patients. The complications were not associated with the surgical approach. Three patients in each group appeared to have had intestinal microperforation missed during surgery, thus requiring relaparotomy performed averagely on postoperative day 2 . All these patients eventually died. A further 3 patients of group II were subjected to relaparotomy due to intestinal obstruction. Intraoperative blood loss of 1,500 $\mathrm{mL}$ was observed in 6 patients ( 3 in each group). And in 1 case of laparo- 
Table 3. Comparing the main perioperative and postoperative data depending on the type of operation

\begin{tabular}{|c|c|c|c|}
\hline Parameter & Elective BN $(n=69)$ & Emergent BN $(n=39)$ & $p$ value \\
\hline \multicolumn{4}{|l|}{ Blood loss volume, $\mathrm{mL}$} \\
\hline Laparoscopy & Me 200 (IQR 200-350); 318 $237^{*}$ & $250,300,200^{* *}$ & 0.418 \\
\hline \multicolumn{4}{|l|}{ Length of hospital stay, days } \\
\hline Laparoscopy & Me 8 (IQR 7-9); 9 $\pm 2 *$ & $6,9,7^{* *}$ & 0.596 \\
\hline Laparotomy & Me 9 (IQR 9-11.5); $10 \pm 2 *$ & Me 16.5 (IQR $13.5-20.5) ; 19 \pm 10^{*}$ & $<0.001$ \\
\hline Sepsis before operation, $n(\%)$ & 0 & $9(23.1)$ & $<0.001$ \\
\hline Intraoperative haemorrhage, frequency, $n(\%)$ & $3(4.3)$ & $3(7.7)$ & 0.37 \\
\hline Intestinal injury, frequency, $n(\%)$ & 0 & $6(15.4)$ & 0.002 \\
\hline Relaparotomy, frequency, $n(\%)$ & 0 & $9(23.1)$ & $<0.001$ \\
\hline Lethal outcome in early postoperative period, frequency, $n(\%)$ & 0 & $18(46.2)$ & $<0.001$ \\
\hline
\end{tabular}

BN, bilateral nephrectomy; Me, median. * Quantitative parameters for clarity are expressed as medians with IQRs and means with standard deviation. ** Three patients corresponded to the indicated characteristics.

scopic BN, we opened the pleural sinus because of a pronounced commissural-cicatricial process in the area of the upper pole of the kidney wherein a large cyst with purulent content was localized.

In our study, $18(16.7 \%)$ patients died in the early postoperative period. Lethal outcomes were not associated with the surgical approach. Studying the thanatogenesis revealed that 9 patients died of progression of preoperative sepsis, 6 patients died resulting from the development of abdominal sepsis due to intestinal injury, and 3 patients died of progressing nosocomial pneumonia. All patients who died had been operated on emergency. The results of comparing the main perioperative and postoperative data depending on the type of operation are shown in Table 3.

The patients operated on emergency had significantly more often received prolonged antimicrobial therapy for infected cysts; 9 patients had sepsis at the time of intervention. Injury of the large intestine and relaparotomy were observed only in patients operated on emergency. Emergency operations were also associated with greater intraoperative blood loss and longer hospital stay.

Univariate analysis of postoperative death risk demonstrated that statistically significant and relatively strong correlations were obtained for the existing at the time of operation sepsis, prolonged, for more than 2 weeks, antibacterial therapy, intestinal injury, and relaparotomy (shown in Table 4). Analysing lethality by means of the binary logistic regression model demonstrated that significant predictors of a lethal outcome were prolonged, for more than 2 weeks, preoperative antibacterial therapy
(AOR 7.5; 95\% CI 1.9-28, $p=0.003$ ) and the CRP level (AOR 1.01; 95\% CI 1.008-1.022, $p<0.001$ ). Assessing the dependence of probability of lethal outcome on the CRP level with the help of ROC analysis, the following curve was obtained (shown in Fig. 1).

The area under the ROC curve amounted to $0.82 \pm$ 0.057 (95\% CI 0.70-0.93), the prognostic model was statistically significant $(p<0.001)$. The value of the CRP level at the cut-off point amounted to $173 \mathrm{mg} / \mathrm{L}$. Patients with CRP levels higher than $173 \mathrm{mg} / \mathrm{L}$ were supposed to have elevated risk of a lethal outcome after the operation. With CRP levels below $173 \mathrm{mg} / \mathrm{L}$, the risk of death was considered insignificant. Sensitivity and specificity amounted to 83 and $83 \%$, respectively. The relationship between the CRP value and length of hospital stay evaluated with the help of Spearman's rank correlation coefficient was statistically significant, of moderate strength $\left(r_{x y}=0.35, p<0.001\right)$.

Histological examination revealed incidental renal cancer in 7 (6.4\%) patients, with no cases of bilateral cancer. Of these, $5(71.4 \%)$ patients had clear-cell renal-cell carcinoma and $2(29.6 \%)$ patients had papillary carcinoma.

\section{Discussion}

Open $\mathrm{BN}$ for $\mathrm{ADPKD}$ is historically considered to be an operation associated with a large number of complications and high risk of a lethal outcome $[2,3]$. Contemporary publications dedicated to $\mathrm{BN}$ evaluate, as a rule, the 
Table 4. Assessment of risk factors for postoperative mortality

\begin{tabular}{|c|c|c|c|c|c|c|c|}
\hline \multirow[t]{3}{*}{ Risk factor } & \multicolumn{4}{|c|}{ Lethal outcome } & \multirow[t]{3}{*}{$p$ value } & \multirow[t]{3}{*}{ OR, 95\% CI } & \multirow{3}{*}{$\begin{array}{l}\text { Cramer's } \\
V\end{array}$} \\
\hline & \multicolumn{2}{|c|}{ presence of the factor } & \multicolumn{2}{|c|}{ no-factor condition } & & & \\
\hline & abs & $\%$ & abs & $\%$ & & & \\
\hline Long-term antibacterial therapy ( 2 weeks and more) & 12 & 66.7 & 6 & 33.3 & 0.006 & $4.67 ; 1.59-13.7$ & 0.28 \\
\hline Sepsis & 9 & 50 & 9 & 50 & $<0.001$ & * & 0.674 \\
\hline Intraoperative haemorrhage & 3 & 16.7 & 15 & 83.3 & 0.057 & $5.8 ; 1.07-31.5$ & - \\
\hline Intestinal injury & 6 & 33.3 & 12 & 66.7 & $<0.001$ & $*$ & 0.542 \\
\hline Relaparotomy & 9 & 50 & 9 & 50 & $<0.001$ & $*$ & 0.674 \\
\hline Open BN & 15 & 83.3 & 3 & 16.7 & 0.169 & $2.89 ; 0.78-10.7$ & 0.158 \\
\hline Laparoscopic BN & 3 & 16.7 & 15 & 83.3 & 0.169 & $0.35 ; 0.09-1.3$ & 0.158 \\
\hline
\end{tabular}

BN, bilateral nephrectomy. ${ }^{*}$ The odds ratio cannot be calculated due to division by 0 .

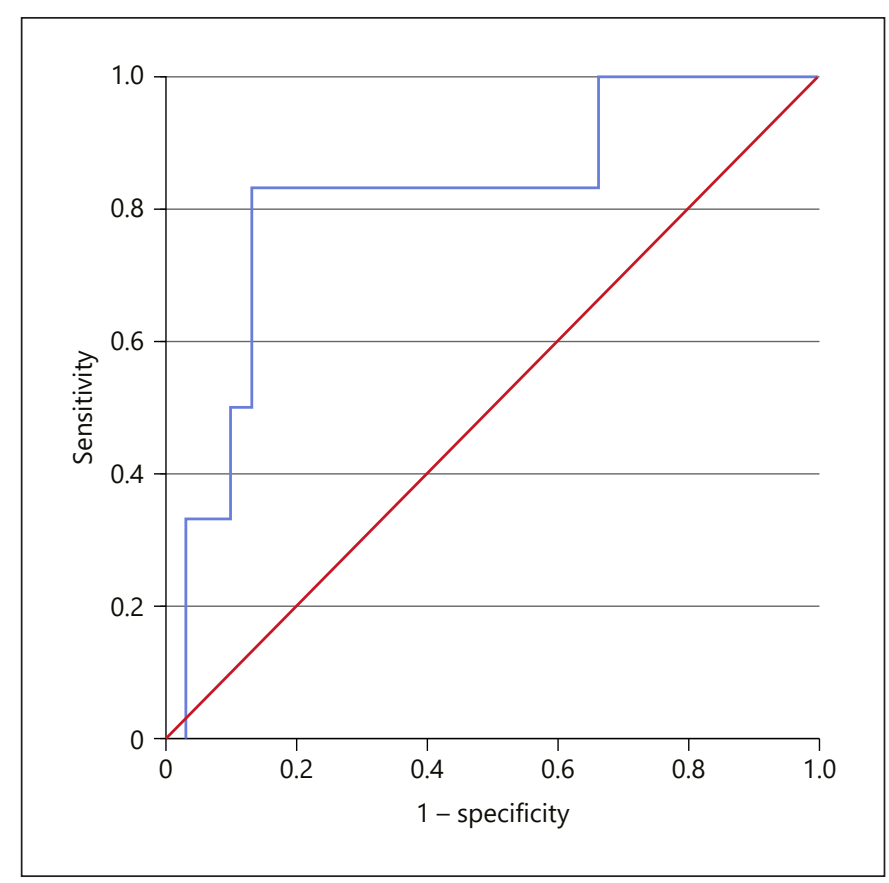

Fig. 1. ROC curve characterizing dependence of probability of postoperative death on the CRP level.

results of the laparoscopic approach, as well as address the problem of when, for whom, and to what extent (unilateral/bilateral) an operation should be performed once kidney transplantation is envisaged. Currently, there seem to be no large studies devoted to open $\mathrm{BN}$, comparing it with the laparoscopic approach. The available literature sources contain only 1 meta-analysis published in 2015 and including 195 cases (laparoscopic BN - 118, open $\mathrm{BN}$ - 77) from 6 studies [5]. This encouraged us to carry out the present study.

The overwhelming majority of publications report increased volume of blood loss and the need for haemotransfusions during open $\mathrm{BN}[5,12,16]$. This fact is especially undesirable in patients awaiting elective kidney transplantation since blood transfusion can cause sensitization $[17,18]$. In our study, despite the fact that the difference of blood loss volume was significantly higher for open $\mathrm{BN}$, it was not substantial and hence there appeared no need for haemotransfusion. Probably, inconsiderable blood loss was accounted for by a series of reasons: first, these operations were performed by the same team of surgeons, and second, we have always been using electrosurgical instruments at all stages of the intervention starting from formation of the approach and ending with transection of the kidney pedicle.

One of disadvantages of laparoscopic BN for ADPKD is the duration of the operation known to be considerably longer compared with that of open $\mathrm{BN}$, which was demonstrated in a series of previously published reports [12, $13,16]$. Increased duration of the operation in laparoscopic approach is attributable to, inter alia, the time necessary to rotate the patient, which is not required when performing midline laparotomy. In our study, it averagely took $20 \pm 10 \mathrm{~min}$ to flip the patient. Despite longer duration of laparoscopic intervention, this method makes it possible to reduce the length of hospital stay; however, the duration of hospital stay after elective operations insignificantly differed depending on the approach. The duration of hospital treatment after laparoscopic $\mathrm{BN}$ in our work turned out to be twice as long as in previous studies reporting an average length of 4 days $[5,12,19]$. We believe that this is because in our practice, early postopera- 
tive rehabilitation of patients is carried out in a hospital rather than at home.

The frequency of lethal outcomes (16.7\%) was relatively high, significantly exceeding that in previously published works, reporting it to be not higher than $5 \%[2,3]$. Analysing lethality, we determined that the surgical approach was significantly not associated with the outcome. The risk factors for postoperative lethality were sepsis having developed prior to surgery, intestinal perforation, and subsequent relaparotomy. The risk of sepsis caused by urinary tract infection in patients with ESKD is considerably higher than in general population $[20,21]$. In our study, 9 (50\%) patients who died had had severe sepsis at the time of the operation caused by infected cysts of both kidneys. Large bowel injury and development of peritonitis and abdominal sepsis resulted in a lethal outcome in $100 \%$ of cases. We believe that a $100 \%$ mortality rate was primarily due to untimely diagnosis of intestinal microperforation, as well as the presence of a favourable background for progression of abdominal infection in conditions of immunosuppression induced by previously performed operation, ESKD [22]. In 3 cases, lethal outcomes were caused by nosocomial pneumonia having led to sepsis. These data once again confirm the fact that dialysis is a risk factor for a lethal outcome in pneumonia [23].

Overall, the risk of lethal outcome after $\mathrm{BN}$ was associated with the patient's state at the time of surgery, severity of SIRS, as well as iatrogenic intestinal injury. Proceeding from this, it can be concluded that in patients with ESKD and multifocal, bilateral infected renal cysts, it is necessary to minimize the duration of antibacterial therapy and perform BN before the development of severe SIRS, with the need to minimize the risk of large intestinal injury being absolutely apparent. We are supporters of surgical treatment of patients with ESKD and also multiple infected renal cysts. This is accounted for by a series of reasons: the choice of antibacterial drugs is limited because only fat soluble antibiotics create optimal concentrations inside the cyst [24]; increased resistance of the most prevalent causative agent Escherichia coli to fluoroquinolones has been reported [25]; there are data on poor penetration of carbapenems into cysts [26]; and adequate transcutaneous drainage of multiple cysts in the majority of cases is impossible. Intestinal injury occurred only in patients operated on emergency. This complication was exceptionally due to the presence of multiple infected cysts, involvement of the paranephrium, and paracolon into the inflammatory process.

Despite the fact that patients with transplanted kidneys $(n=12)$ were immunosuppressed, we observed no infectious complications after surgery. Probably, on the one hand, this was because they had no SIRS at the time of intervention, and on the other hand, in all cases laparoscopic $\mathrm{BN}$ was performed. However, to draw a more reliable conclusion concerning the effect of immunosuppression on the therapeutic outcome depending on the variant of the surgical access will require a larger patient cohort and a comparison group (open operation), which was not the case in our work.

In our series, histological examination revealed incidental renal cancer in 7 (6.4\%) patients, which is consistent with previously published works and confirms a higher frequency of renal cancer in patients with ADPKD and ESKD compared to general population. Jilg et al. [27] reported that incidental renal tumours were revealed in $5.3 \%$ of cases (16 malignant lesions in 301 kidneys). Hajj et al. [28] reported a higher prevalence of $12.4 \%$ (11 of 89 kidneys were diagnosed with carcinomas).

Within the framework of this study, we did not evaluate the requirements for narcotic analgesics after the operation depending on the surgical approach because according to the protocols of our institution, virtually all patients in the early postoperative period for $72 \mathrm{~h}$ received continuous epidural administration of ropivacaine at a dose of $2 \mathrm{mg} / \mathrm{mL}$. Neither did we assess the approachdependent need for blood transfusion since only 6 (5.6\%) patients required blood transfusion due to intraoperative haemorrhage.

This work had significant limitations. It was a retrospective and single-centre study. The choice of the operation (open or laparoscopic) was mainly determined by the type of the operation (elective or emergent).

\section{Conclusion}

Overall, the results of open BN are non-inferior to those of laparoscopic BN. The frequency of complications, lethal outcomes, and the volume of intraoperative blood loss did not depend on the surgical approach. Significant advantages of the laparoscopic approach include a shorter postoperative scar, rapid rehabilitation of patients, and decreased length of hospital stay. Nevertheless, we suppose that for patients with significantly enlarged kidneys requiring formation of a large access, it is possible to consider a variant of open surgical treatment. Patients with sepsis, SIRS on the background of infected renal cysts, with CRP levels above $173 \mathrm{mg} / \mathrm{L}$ have a high probability of death after $\mathrm{BN}$. Undiagnosed large bowel injury is associated with an extremely high risk of postoperative death. 


\section{Acknowledgements}

The authors thank all the assistants who participated in the study.

\section{Statement of Ethics}

All patients that were included in this research work gave their written informed consent and the study protocol was approved by the institute's committee on human research.

\section{Conflict of Interest Statement}

The authors have no conflicts of interest to declare.

\section{Funding Sources}

Sechenov University.

\section{Author Contributions}

All authors have contributed in writing and reviewing the manuscript.

\section{References}

1 Desai PJ, Castle EP, Daley SM, Swanson SK, Ferrigni RG, Humphreys MR, et al. Bilateral laparoscopic nephrectomy for significantly enlarged polycystic kidneys: a technique to optimize outcome in the largest of specimens. BJU Int. 2008;101(8):1019-23.

2 Bennett AH, Stewart W, Lazarus JM. Bilateral nephrectomy in patients with polycystic renal disease. Surg Gynecol Obstet. 1973;137(5): 819-20.

3 Mendelssohn DC, Harding ME, Cardella CJ, Cook GT, Uldall PR. Management of endstage autosomal dominant polycystic kidney disease with hemodialysis and transplantation. Clin Nephrol. 1988;30(6):315-9.

4 Chen K, Tan YG, Tan D, Pek G, Huang HH, Sim SPA. Predictors and outcomes of laparoscopic nephrectomy in autosomal dominant polycystic kidney disease. Investig Clin Urol. 2018;59(4):238-45.

5 Guo P, Xu W, Li H, Ren T, Ni S, Ren M. Laparoscopic nephrectomy versus open nephrectomy for patients with autosomal dominant polycystic kidney disease: a systematic review and meta-analysis. PLoS One. 2015;10:1-13.

6 Lipke MC, Bargman V, Milgrom M, Sundaram CP. Limitations of laparoscopy for bilateral nephrectomy for autosomal dominant polycystic kidney disease. J Urol. 2007;177(2):627-31.

7 Ivey BS, Lucas SM, Meyer CA, Emley TE, Bey A, Gardner TA, et al. Conversions in laparoscopic renal surgery: causes and outcomes. J Endourol. 2011;25(7):1167-73.

8 Whitten MG, Van Der Werf W, Belnap L. A novel approach to bilateral hand-assisted laparoscopic nephrectomy for autosomal dominant polycystic kidney disease. Surg Endosc. 2006;20(4):679-84.

9 Bendavid Y, Moloo H, Klein L, Burpee S, Schlachta CM, Poulin EC, et al. Laparoscopic nephrectomy for autosomal dominant polycystic kidney disease. Surg Endosc. 2004; 18(5):751-4.

10 Lee DI, Clayman RV. Hand-assisted laparoscopic nephrectomy in autosomal dominant polycystic kidney disease. J Endourol. 2004; 18(4):379-82.

11 Dunn MD, Portis AJ, Elbahnasy AM, Shalhav AL, Rothstein M, McDougall EM, et al. Laparoscopic nephrectomy in patients with endstage renal disease and autosomal dominant polycystic kidney disease. Am J Kidney Dis. 2000;35(4):720-5.

12 Eng M, Jones CM, Cannon RM, Marvin MR. Hand-assisted laparoscopic nephrectomy for polycystic kidney disease. JSLS. 2013;17(2): 279-84.

13 Mak DKC, Ilie CP, Abedin A, Gommersall L, Luscombe C, Golash A. Bilateral hand-assisted laparoscopic nephrectomy in adult polycystic kidney disease patients: a UK centre experience. J Med Life. 2012;5:226-31.

14 American College of Chest Physicians/Society of Critical Care Medicine Consensus Conference: definitions for sepsis and organ failure and guidelines for the use of innovative therapies in sepsis. Crit Care Med. 1992;20: 864-74.

15 Rea LM, Parker RA. Designing and conducting survey research. San Francisco: JosseyBoss; 1992. p. 203.

16 Verhoest G, Delreux A, Mathieu R, Patard JJ, Vigneau C, Rioux-Leclercq N, et al. Transperitoneal laparoscopic nephrectomy for autosomal dominant polycystic kidney disease. ISLS. 2012;16(3):437-42.

17 Scornik JC, Ireland JE, Howard RJ, Pfaff WW. Assessment of the risk for broad sensitization by blood transfusions. Transplantation. 1984; 37(3):249-53

18 Ghio M, Contini P, Mazzei C, Brenci S, Barberis G, Filaci G, et al. Soluble HLA class I, HLA class II, and Fas ligand in blood components: a possible key to explain the immunomodulatory effects of allogeneic blood transfusions. Blood. 1999;93(5): $1770-7$.

19 Binsaleh S, Luke PP, Nguan C, Kapoor A. Comparison of laparoscopic and open nephrectomy for adult polycystic kidney dis- ease: operative challenges and technique. Can J Urol. 2006;13(6):3340-5.

20 Sakhuja A, Nanchal RS, Gupta S, Amer H, Kumar G, Albright RC, et al. Trends and outcomes of severe sepsis in patients on maintenance dialysis. Am J Nephrol. 2016;43(2):97103.

21 Sarnak MJ, Jaber BL. Mortality caused by sepsis in patients with end-stage renal disease compared with the general population. Kidney Int. 2000;58(4):1758-64.

22 Galli F. Protein damage and inflammation in uraemia and dialysis patients. Nephrol Dial Transplant. 2007;22(Suppl 5):v20-36.

23 Li G, Cook DJ, Thabane L, Friedrich JO, Crozier TM, Muscedere J, et al. Risk factors for mortality in patients admitted to intensive care units with pneumonia. Respir Res. 2016; 17(1):80-9.

24 Suwabe T, Araoka H, Ubara Y, Kikuchi K, Hazue R, Mise K, et al. Cyst infection in autosomal dominant polycystic kidney disease: causative microorganisms and susceptibility to lipid-soluble antibiotics. Eur J Clin Microbiol Infect Dis. 2015;34(7):1369-79.

25 Allocati N, Masulli M, Alexeyev MF, Di Ilio C. Escherichia coli in Europe: an overview. Int $J$ Environ Res Public Health. 2013;10:6235-54.

26 Hamanoue S, Suwabe T, Ubara Y, Kikuchi K, Hazue R, Mise K, et al. Cyst infection in autosomal dominant polycystic kidney disease: penetration of meropenem into infected cysts. BMC Nephrol. 2018;19(1):272.

27 Jilg CA, Drendel V, Bacher J, Pisarski P, Neeff H, Drognitz O, et al. Autosomal dominant polycystic kidney disease: prevalence of renal neoplasias in surgical kidney specimens. Nephron Clin Pract. 2013;123(1-2): $13-21$.

28 Hajj P, Ferlicot S, Massoud W, Awad A, Hammoudi Y, Charpentier B, et al. Prevalence of renal cell carcinoma in patients with autosomal dominant polycystic kidney disease and chronic renal failure. Urology. 2009;74(3): 631-4. 